\title{
The Tesla Currents in Electrodynamics
}

\author{
Andrew Chubykalo ${ }^{1}$ \& Viktor Kuligin ${ }^{2}$ \\ ${ }^{1}$ México Unidad Académica de Física, Universidad Autónoma de Zacatecas, A.P. C-580, Zacatecas \\ ${ }^{2}$ Physical faculty Department of an electronics, Voronezh State University, Russia \\ Correspondence: Andrew Chubykalo, México Unidad Académica de Física, Universidad Autónoma de Zacatecas, \\ A.P. C-580, Zacatecas. E-mail: achubykalo@yahoo.com.mx
}

Received: August 8, 2018

Accepted: September 20, 2018

Online Published: September 27, 2018

doi:10.5539/apr.v10n5p79

URL: https://doi.org/10.5539/apr.v10n5p79

\begin{abstract}
The paper theoretically shows that the Maxwell equations in the Lorentz gauge deal with not only inertial charged particles, but also charged particles that do not have inertia (virtual charges). Virtual charges appear on the surface of metals. Their movement is the currents of Tesla. Experiments confirming their existence are presented, and some features that reveal them. The influence of virtual currents on the process of transfer of conduction electrons in $p-n$ junctions of semiconductor devices is especially interesting. The results obtained can change our understanding of phenomena in the microcosm.
\end{abstract}

Keywords: Virtual charges, conduction electrons, Avramenko plug, Tesla currents

\section{Introduction}

There are not many physicists who will argue that Maxwell's equations describe all phenomena of electromagnetism without exception. Electrodynamics cannot be considered a complete theory. In electrodynamics, there are little studied phenomena. In this paper, we consider the problem of energy transfer by one wire. The effect, discovered by Avramenko (Avramenko, Lisin, \& Zaev, 1991), has existed for a long time, the scientists, however, still could not give an adequate explanation of this phenomenon.

\section{Charges and Currents on the Metal Surface}

Tesla was an ingenious experimenter. However, his experiments were not supported by equations. Therefore, the Tesla currents have a mystery to this day. Many experimenters closely approached the possibility of repeating Tesla's experiments. One of the researchers was Avramenko, who conducted a number of interesting experiments, transmitting electric energy through one wire.

The problem of explaining the Avramenko effect is not simple, so we will start from afar. In electrodynamics, the boundary conditions for the fields at the interface of two media are strictly deduced. We are interested only in conductors, so we will write down the boundary conditions for the electric and magnetic fields for the conductor surface. When the fields act on the surface of an ideal conductor, surface charges and currents $\left(\varrho_{\text {surf }} ; \mathbf{j}_{\text {surf }}\right)$ arise that prevent the penetration of the fields into the metal.

$$
\mathbf{j}_{\text {surf }}=-(\mathbf{n} \times \mathbf{H}) \text { and } \varrho_{\text {surf }}=\varepsilon(\mathbf{n} \cdot \mathbf{E}),
$$

where $\mathbf{E}$ is the electric field strength on the metal surface, $\mathbf{H}$ is the intensity of the magnetic field on the metal surface, $\mathbf{n}$ is the unit normal to the surface, $\varrho_{\text {surf }}$ and $\mathbf{j}_{\text {surf }}$ are surface charge density and surface current densities.

When explaining boundary conditions and surface phenomena, there is a question that is not usually considered in textbooks. Assume that electromagnetic or light waves hit the surface of the metal (Landau \& Lifshitz, 2010). Suppose that the metal surface reflects electromagnetic or light waves. Then the electromagnetic fields change very quickly. What processes take place on the metal surface? Are the boundary conditions satisfied almost instantly? The authors (Avramenko, Lisin, \& Zaev, 1991) avoid a direct answer to this question. They usually refer to "conduction electrons". However, the conduction electrons have a large inertia. So this explanation is rather controversial.

The fields $\mathbf{E}$ and $\mathbf{H}$ are retarded. Consequently, the surface currents and charges in formulas (1) are also retarded. Like the fields $\mathbf{E}$ and $\mathbf{H}$, the surface charges must satisfy the wave equation, and they can move at the speed of 
light. These charges have no inertia, unlike electrons. This is a new type of classical surface conductivity of metals. Physicists are constantly confronted with these charges, but they did not fully investigate them. Because of the lack of inertia, the boundary conditions (1) are satisfied almost instantaneously. Using Maxwell's equations, we analytically show this in the article below. We also give the results of some experiments. Probably, surface currents and charges will be included in the system of equations of electrodynamics in the future. The standard classical electronic theory of conductivity deals only with inertial charges (electrons). Therefore, it is limited.

We will not consider the zone theory. Let us try to give an explanation from the standpoint of classical physics, especially since we are considering macroscopic phenomena.

1) We know that when a current flows in a conductor, the average velocity of the ordered motion of the conduction electrons is millimeters per second. This is a very small speed for fulfilling the boundary conditions.

2) In order to have a high speed, the electron must dial it under the action of an external field. The inertia of the electron impedes this process, making it difficult to quickly change the magnitude and direction of the velocity under the action of external fields. Thus, attempts to explain the fulfillment of boundary conditions at a surface acted upon by electromagnetic waves with classical boundary conditions with the aid of conduction electrons do not stand up to criticism.

Let us start with the basics. Any conductor is a quasi-neutral system. To have an electric field around the insulated conductor, it is necessary that there be excess charges on the surface of the conductor. They violate the quasineutrality condition of the system. The electric field does not penetrate the conductor. Therefore, the surface of a charged conductor is an equipotential, i.e. surface of the same potential. We assume that the potential of an infinitely distant surface is equal to zero. Then an isolated charged body can be considered as the own capacitance of an isolated conductor. The connection between the potential of an isolated solitary conductor is described by the formula $q=C \varphi,\left(\varphi_{\infty}=0\right)$, where $C$ is the capacity of the surface

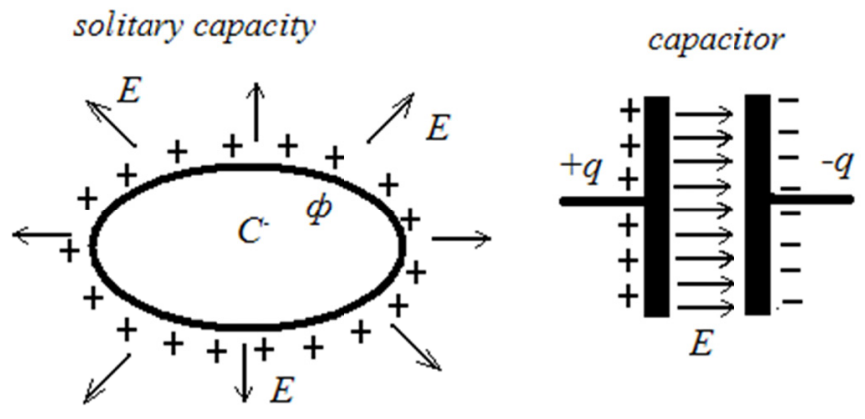

Figure 1. Capacities and excess charges

Due to the inertial properties of the electrons we put forward the hypothesis that excess positive and negative charges are not an excess or a lack of conduction electrons in a conductor. These are special charges capable of changing their state of motion much faster than electrons, that exist along with electrons, positrons, protons, etc. To illustrate this, consider the propagation of a short electromagnetic pulse in a coaxial cable (see Fig. 2).

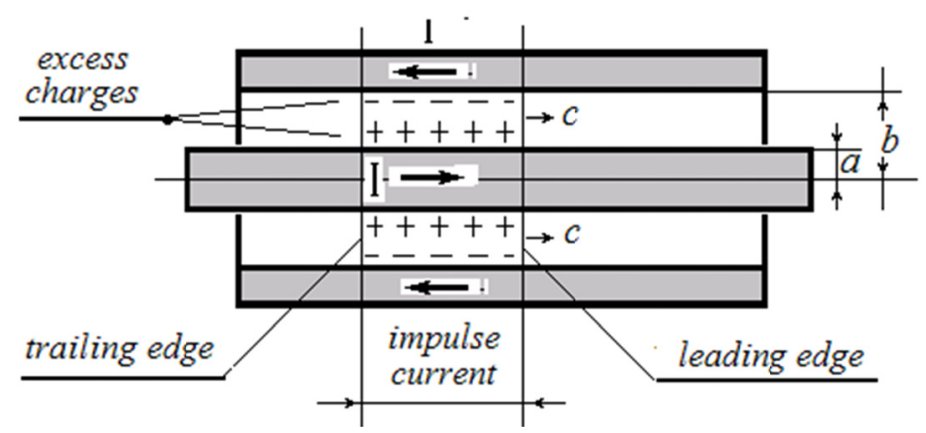

Figure 2. Propagation of an electromagnetic pulse along a coaxial line 
Inside the coaxial cable fields $\mathrm{E}$ and $\mathrm{H}$ are created by moving excess positive and negative charges (Landau \& Lifshitz, 2010). Let the pulse propagate along the $z$ axis. Let us calculate some quantities:

1) The charge on the elementary segment $d z$ of the outer coaxial cylinder is $d q_{1}=2 \pi b \varepsilon E_{r}(b) d z$; where $E_{r}(b)$ is the electric field at $r=b$.

2) The charge on the elementary segment $d z$ of the interior coaxial cylinder is $d q_{2}=2 \pi a \varepsilon E_{r}(a) d z$; where $E_{r}(a)$ is the electric field at $r=a$. Hence $E_{r}(r)=I_{1} / r$.

Obviously, the law of charge conservation holds: $\left|d q_{1}\right|=\left|d q_{2}\right|=d q$. We calculate the values of the surface currents in these conductors.

3) External coaxial cylinder: $\mathrm{I}_{1}=2 \pi b \mathrm{H}_{\varphi}(\mathrm{b})$.

4) Internal coaxial cylinder: $\mathrm{I}_{2}=2 \pi a \mathrm{H}_{\varphi}(\mathrm{a})$. Hence $\mathrm{H}_{\varphi}(\mathrm{r})=\mathrm{I}_{2} / \mathrm{r}$.

The surface currents of these conductors are, respectively, $\left|I_{1}\right|=\left|I_{2}\right|=I$ Now we can easily calculate the rate of movement of excess charges, for example, for an internal conductor of a coaxial line. On the one hand, we have:

$$
\mathrm{W} I=\frac{d q}{d t}=\frac{d q}{d z} \frac{d z}{d t}=v \frac{d q}{d z}=v 2 \pi a \varepsilon E_{r}(a),
$$

on the other hand:

$$
I=2 \pi a H_{\varphi}(a)
$$

Comparing these expressions and taking into account that $E_{r}(a) / H_{\varphi}(a)=\sqrt{\mu / \varepsilon}$ we get: $v=c$. Try to make the "free" conduction electron move at a similar speed! But in waveguides the phase velocity of excess charges exceeds the speed of light in a vacuum!

So, the excess charges in the coaxial line move with the speed of light! This is one of the important points. Another point is that positive and negative excess charges are not born in pairs, but separately, ignoring the law of conservation of charge. Therefore, we will call such charges virtual charges. It is the virtual charges that are mainly responsible for the instantaneous fulfillment of the boundary conditions on the surface of the conductors.

\section{Virtual Charges (Or: Surface Charges Without Inertia)}

Let the electromagnetic wave fall on the surface of the conductor. We write the boundary conditions.

$$
\mathbf{j}_{\text {surf }}=-[\mathbf{n} \times \mathbf{H}] \text { and } \varrho_{\text {surf }}=\varepsilon(\mathbf{n} \cdot \mathbf{E}) .
$$

The wave excites surface currents and charges. On the one hand, the current density $\mathbf{j}_{\text {surf }}$ satisfies the continuity equation

$$
\operatorname{div} \mathbf{j}_{\text {surf }}=-(\mathbf{n} \cdot \operatorname{rot} \mathbf{H})=-\varepsilon \frac{\partial(\mathbf{n} \cdot \mathbf{E})}{\partial t}=-\frac{\partial \varrho_{\text {surf }}}{\partial t} .
$$

On the other hand, we have on the surface of an ideal conductor only a common electric field $\mathbf{E}$ directed along the normal to the surface. Therefore, we can write:

$$
\begin{gathered}
\frac{\partial \mathbf{j}_{\text {surf }}}{\partial t}=-\frac{1}{\mu}\left[\mathbf{n} \times \frac{\partial(\mu \mathbf{H})}{\partial t}\right]=-\frac{1}{\mu}[\mathbf{n} \times \operatorname{rot} \mathbf{E}] \\
=-\frac{1}{\mu \varepsilon} \operatorname{grad} \varepsilon(\mathbf{n} \cdot \mathbf{E})=-c^{2} \operatorname{grad} \varrho_{\text {surf }} .
\end{gathered}
$$

Since we are dealing with rapidly changing phenomena, it follows from Eq. (3.2) that

$$
\frac{\partial \operatorname{rot} j_{\text {surf }}}{\partial t}=\operatorname{rot}\left(-c^{2} \operatorname{grad} \varrho_{\text {surf }}\right)=0 .
$$

Combining equations (3.1) and (3.2) and taking into account (3.2a) we finally obtain:

$$
\begin{gathered}
\Delta \mathbf{j}_{\text {surf }}-\frac{\partial^{2} \mathbf{j}_{\text {surf }}}{c^{2} \partial t^{2}}=0, \\
\Delta \mathbf{j}_{\text {surf }}-\frac{\partial^{2} \mathbf{j}_{\text {surf }}}{c^{2} \partial t^{2}}=0 .
\end{gathered}
$$

So, surface charges and currents satisfy homogeneous wave equations. Let $\xi$ and $\eta$ are coordinates of a surface element and $r=\sqrt{\xi^{2}+\eta^{2}}$. Then the solutions of these equations will be

$$
\mathbf{j}_{\text {surf }}=\mathbf{c} \varrho_{\text {surf } 1}(r-c t)+\mathbf{c} \varrho_{\text {surf } 2}(r+c t),
$$


and

$$
\varrho_{\text {surf }}=\varrho_{\text {surf1 }}(r-c t)+\varrho_{\text {surf2 }}(r+c t),
$$

where $\mathbf{c}$ is the velocity vector (the speed of light in a vacuum).

We note the obvious properties of virtual charges (or surface charges without inertia):

- We see that virtual charges propagate at the speed of light along the surface of an ideal conductor. They do not have an inertial mass.

- Virtual charges arise (are excited) and are destroyed due to the influence of external electric and magnetic fields. Obviously, the law of conservation of charge does not hold for them.

- Virtual charges realize the phenomenon of "superconductivity" on the surface of conductors at room temperature. This is a new kind of conductivity, the description of which is not in the Maxwell equations. However, virtual charges and currents appear when the boundary conditions on the metal surface are formulated.

- We assume that virtual charges can interact with conduction electrons and transfer some energy to them or take their energy.

- We do not know the nature of virtual charges. A macroscopic version of their description is absent in quantum electrodynamics as well. Perhaps there is some kind of neutral environment enveloping the particles, which is "charged and discharged" when exposed to external fields.

Let us now turn to the description of experimental studies related to virtual charges.

\section{The Experiment of Avramenko}

Now we can discuss the experiment of Avramenko, Lisin, and Zaev (1991). So, on 5.08 .90 an experiment was demonstrated in one of the Moscow Power Engineering Institute (MPEI) laboratories, the scheme of which is shown in Fig. 3.

The experimental setup included a machine generator 1 with a power of up to $100 \mathrm{~kW}$, generating a voltage with a frequency of $8 \mathrm{kHz}$. Note: This machine generator supplied power to the step-up transformer 2. It is possible to use a Tesla transformer instead of a step-up transformer.

One terminal of the secondary winding was free (not connected to anything, as shown in Fig. 3). To the second terminal, the following elements were connected in series: thermoelectric milliampermeter 3 , thin tungsten wire 4 (wire length $2.75 \mathrm{~m}$, diameter $15 \mu \mathrm{m}$ ) and "Avramenko plug" 5.

The Avramenko plug is a closed loop containing two series-connected diodes in which the common point is connected to the previously described circuit and the load. The load was 6 parallel incandescent bulbs. Through this single-wire circuit Avramenko was able to transfer from the generator to the load (incandescent lamps) an electrical power of about $1300 \mathrm{~W}($ !). The electric bulbs shone brightly.

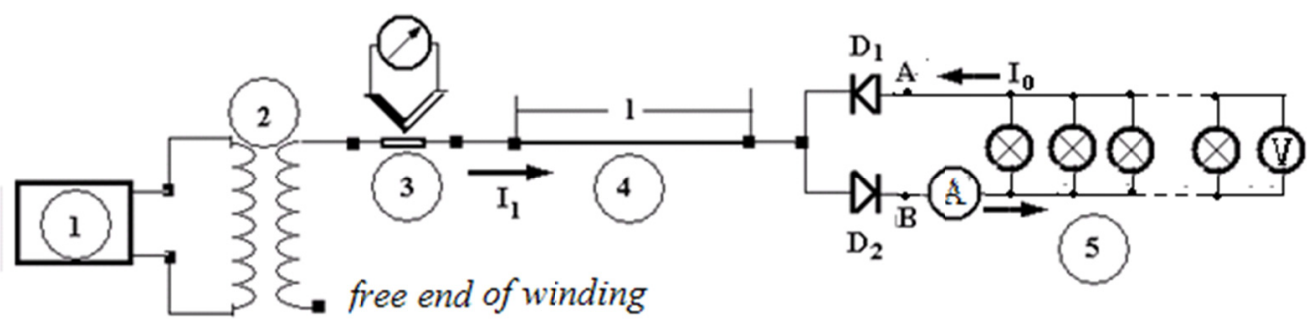

Figure 3. Scheme of the experiment of Avramenko

It would seem that this phenomenon is easily explicable. However, with a detailed explanation of the phenomenon, difficulties arose. The current $I_{0}$ in the Avramenko plug is 6A. It would seem that the current $I_{1}$ in the circuit connecting the end of the winding of the Tesla transformer to the Avramenko plug should be close to or approximately equal to the current $I_{0}$. However, the thermoelectric milliammeter 3 fixed a very small value of the current $I_{1}\left(I_{1}=2 \mathrm{~mA}(!)\right)$, and the thin tungsten wire 4 did not even heat up! This circumstance served as the main reason for the difficulty in explaining the results of Avramenko's experiment. 
Let us give some conclusions made by Avramenko and his colleagues on the basis of studies in Avramenko, Lisin, and Zaev (1991) and Zaev (1994).

1) The current Io in the Avramenko plug increases linearly with increasing frequency (measuring range 5-100 $\mathrm{kHz}$ ) and practically linearly increases with increasing generator voltage at a constant frequency. This indicates the capacitive nature of the electrical circuit.

2) The magnetic field and Ohm's law in the conductor connecting the Avramenko plug with the generator was not detected.

3) The current $I_{1}$ was very small in comparison with the current $I_{0}$ and was practically not detected either by the thermal or magnetoelectric current meter. For this reason, the series resistors (up to several tens of $M \Omega$ ), capacitors and inductances in the connecting circuit (Tesla's transformer - Avramenko plug) had an extremely small attenuating effect on the $I_{0}$ current in the Avramenko's plug.

4) In the Avramenko plug, this current is converted into a conventional pulsating current $I_{0}$. However, in this explanation, not everything is clear. If we measure the voltage $U$ at the points $\mathrm{AB}$ by an electrostatic voltmeter and the current is a normal ammeter, then when calculating the power in the load, there is a discrepancy with the classical laws. The calculated powers $P_{2}^{\prime}=I_{0}^{2} R ; P_{2}^{\prime \prime}=I_{0} U$, Ohm's law $U=I_{0} R$ (where $R$ is the resistance of the circuit containing the bulbs) did not match. The authors write that the resistors "seem to lose their face values" (Avramenko, Lisin, \& Zaev, 1991).

Later we will comment on some of Avramenko's conclusions.

\section{Search for Ways to Explain}

Avramenko and his colleagues could not explain the experiment. We have conducted special studies. The results will be discussed later.

Let us start with a simple example. Consider the Hertz dipole.

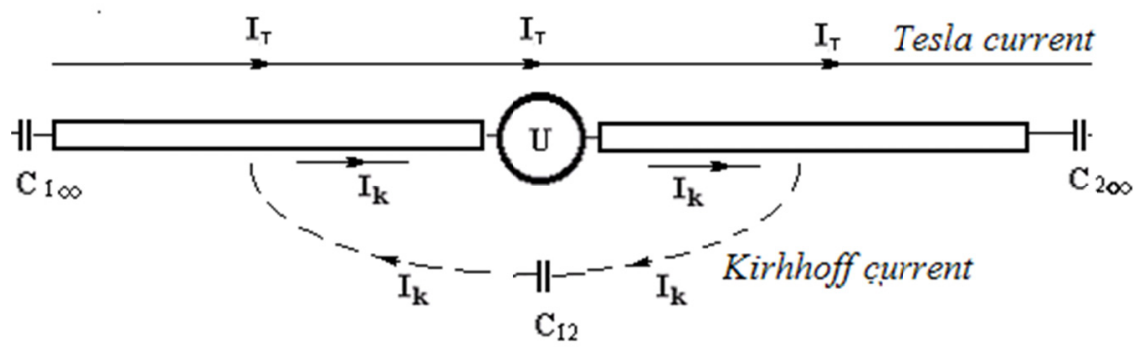

Figure 4. Hertz dipole

Between the "mustaches" of the Hertz dipole, a generator U is inserted. It creates two currents:

1) Kirchhoff's current. This current flows through the closed circuit "mustache 1" - the generator - "mustache 2" - the mutual capacity between the "whiskers" $C_{12}$. Kirchhoff's current is interesting because the current in any cross section of the closed circuit is the same. In this case, the same current $I_{k}$, which depends on time, flows in the Kirchhoff closed circuit. This is the current created by the conduction electrons.

2) Tesla current. This current is formed by virtual charges. Let us describe the circuit: the bias current flowing from the endlessly removed surface to the metal body 1 (solitary capacitance $C_{1 \infty}$ ) - conductor "antenna 1"generator - conductor "antenna(metal body) 2" - displacement current from "antenna (metal body) 2" to the infinitely remote surface (solitary capacity $C_{2 \infty}$ ).

We confine ourselves to the case of low frequencies, when the length of the "mustache" is much smaller than the wavelength. In addition, we will assume that the mutual capacity between the "whiskers" is very small and Kirchhoff's currents can be neglected. This allows you to explore certain phenomena in a "pure form." A diagram suitable for investigation is shown in Fig. 5. 


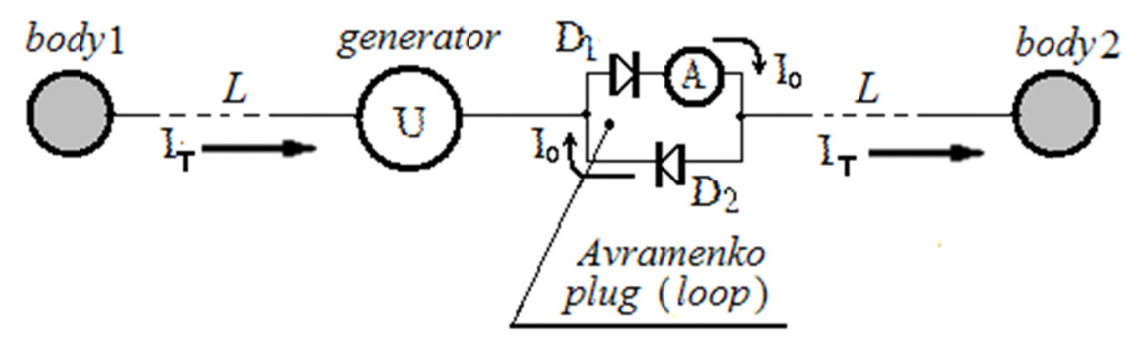

Figure 5. Simplified diagram for analysis

Now let us list those simplifications that allow us to analyze the Avramenko experiment.

1) The Length L between "Body 1" or "Body 2" and the wires of generator are much smaller than the wavelength. This allows us to neglect phenomena associated with the electromagnetic radiation of the system $L \ll \lambda$. If the length of the conductor is of the order of several meters, then the wavelength $\lambda$ at $10 \mathrm{kHz}$ is 30,000 meters.

2) The conductors $L$, connecting the terminals of the generator with each body had a very small diameter, which contributed to a significant decrease in the mutual capacitance of the bodies $C_{12}$. This was also facilitated by the fact that the dimensions of the bodies were very small compared to the distance $L$.

It turns out that for observing phenomena it is possible to use standard devices that do not require the use of a Tesla transformer.

1) Generator GZ-35. Product specifications: G3-35 is generator of audio signals with low level of harmonic distortion. The device is assembled on 8 electron tubes. It generates a sinusoidal signal of $20 \mathrm{~Hz}-200 \mathrm{kHz}$ in 4 subbands with a harmonic coefficient of less than $0.05 \%$ at a load of $600 \Omega$. The maximum output voltage is $8 \mathrm{~V}$.

2) A standard power transformer with an iron core. The transformation ratio is $N_{1} / N_{2}=50: 5000$.

3) The effect was preserved without significant deviations at frequencies up to $200 \mathrm{kHz}$ and depended little on the frequency properties and type of diodes.

4) As an indicator, we used a multi-limit magnetoelectric milliammeter.

\section{Unusual Results}

Here we must note the difficulties encountered by all the experimenters investigating the Tesla currents:

- To date, there is no clear understanding of the nature of virtual charges and charge transfer processes on the metal surface. The theory of Tesla currents is at the initial stage of development.

- For experimental studies, even the most advanced devices are not very suitable. Virtually any measuring device has a large solitary (own) capacity with respect to an infinite sphere. Tesla's currents will always flow along its surface. In addition, most of the measuring devices are electrically connected to the power network.

- Standard methods for conducting such experimental studies have not yet been developed.

- Before we begin to discuss the results of these experimental studies, we will outline some considerations. From our point of view, the explanation of phenomena in the Avramenko experiment (Zaev, 1994) contains doubtful moments:

- We know that the Tesla current in Avramenko's experiment is created by virtual charges. These charges do not have inertia and kinetic energy. They move along the surface of the conductor "without friction", i.e. without loss of energy. Therefore, it is impossible to measure the Tesla current by a thermoelectric milliammeter. The Tesla current is not capable of producing power at resistance. For this reason, we can assume that the real Tesla current can have a magnitude of a few amperes.

- The same reason explains the fact that the inclusion of a few megaohms into the resistance wire in series does not practically affect the measurement results.

- The authors of the experiment claim that they failed to detect a magnetic field. Unfortunately, the experiment did not describe the instruments used to measure the magnetic field. There is also no description of the method for measuring the magnetic field. 
Now we give a description of our experiments. Fig. 6 shows 3 variants of connecting the Avramenko plug to the connecting wire. Variant $a$ is the standard insertion of the plug into a wire break. The Tesla current $I_{T}$ flows in a very small amount to the plug, a pulsating current $I_{0}$, hundreds and thousands of times greater than the current $I_{T}$, flows in the plug loop.
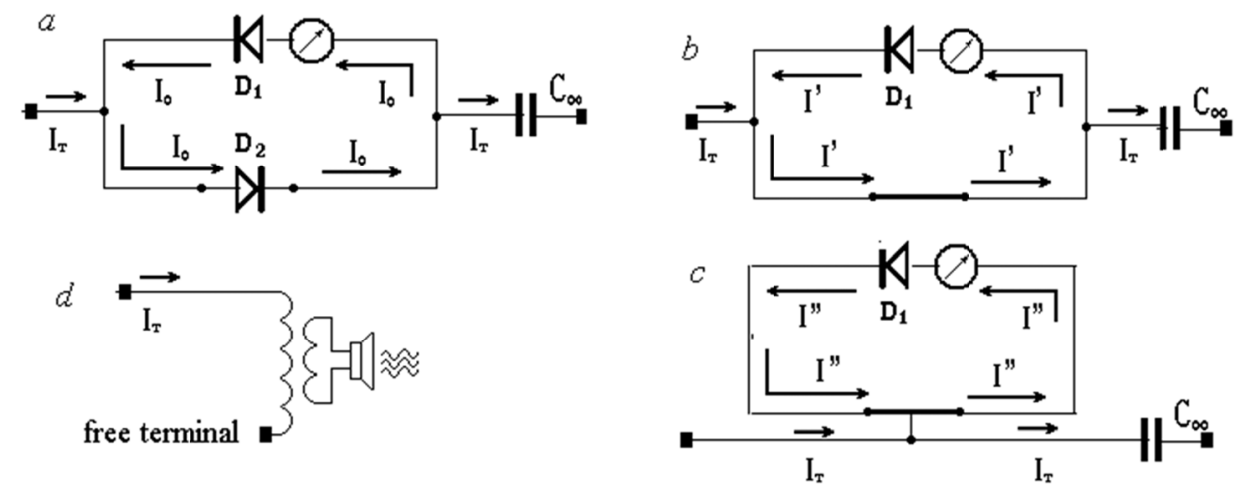

Figure 6

Below is a variant of $b$ for those who wish to test their knowledge in the theory of electrical circuits. In this embodiment, diode $D_{2}$ is replaced by a short-circuited jumper. What is the value of the pulsed current $I^{\prime}$ ? According to the theory of electrical circuits, there should be no pulsating current in the "spoiled" plug of Avramenko. The arrow of the measuring device must remain at zero. However, the device will record the current $I^{\prime}=I_{0} / 2$, i.e. 2 times less! This is an amazing and unexpected result. Surface currents obey other laws.

No less interesting is the variant $c$. In it the plug of Avramenko is excluded from the circuit with the current $I_{T}$. It is connected only by a single conductor with a wire through which the Tesla current flows. And here we are faced with violations of the classical theory of electrical circuits. The current $I^{\prime \prime}$ in the plug exists and is also equal to half the current $I_{0}, I^{\prime \prime}=I_{0} / 2$.

If you look closely, the topological identity variants in Fig. $6(b, c)$ is obvious. As we see, there are no "special devices" apart from the diode in the circuit. Consequently, the whole "highlight" is in the work of the n-p transition of the transistor. Changing the spatial orientation of the contour Fig. 6 (c) did not affect the magnitude of the current. We replaced the Avramenko plug with a step-down transformer with a phone (earphone) included in the secondary winding as shown in Fig. 6 (d). We heard sound vibrations. Hence the Tesla current has a magnetic field!

Scientists must answer the question: how can virtual charges affect the main and non-primary carriers of the n-p junction and create electronic currents in a closed loop without sources (for example, Fig. 6c)?

We draw attention to one more important experimental result. In one of the experiments (see Fig. 3) Avramenko changed the load in the plug, as shown in Fig. 7. Instead of incandescent lamps, an air spark gap and a charging capacitance $C$ were used. Avramenko used high-voltage rectifiers (D1 and D2).

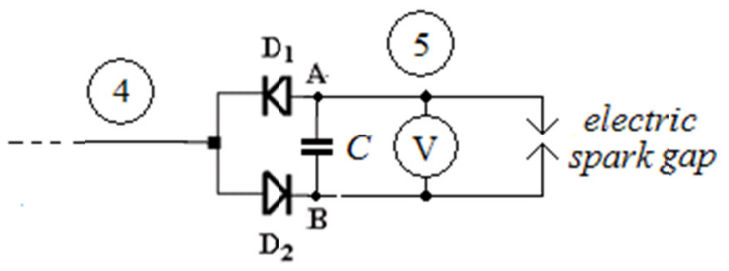

Figure 7

The authors write (Avramenko, Lisin, \& Zaev, 1991): “.... if ... connect the plug to the wire under an alternating voltage of 10-10000 $\mathrm{V}$, the current in the plug loop will circulate in a constant direction, but pulsating in 
magnitude, and soon sparks will scatter from the spark gap P. When? This depends on the capacitance $C$, the ripple frequency and the gap size of the arrester. A voltmeter connected to the spark gap will show a potential difference of up to $10-20 \mathrm{kV}$, or even 100-150 kV."

And again there are interesting questions. The voltage on the capacitor increases as the capacitor is charged. This voltage creates a potential barrier for electrons passing through the diodes and charging the capacitor.

A spark discharge is possible if a potential difference of more than 5000 volts arises between the spark gap electrodes. In experiments, the voltage across the spark gap reached $100-150 \mathrm{kV}$.

How do virtual charges "transfer" the conduction electrons from one capacitor plate to another through diodes, overcoming this voltage? Why at such high voltages on the capacitor $C$ and between the spark gap electrodes does not the phenomenon of reverse breakdown of the $n-p$ junction in diodes occur?

Virtual currents, as we see, have a great specificity that distinguishes them from currents in standard Kirchhoff chains.

\section{Conclusion}

Let us sum up our research:

1) It has been theoretically established that Maxwell's equations deal not only with conduction electrons and other inertial charges. They describe virtual (non-inertial) charges moving along the surface of conductors at the speed of light, forming surface currents (Tesla currents).

2) The existence of virtual charges and Tesla currents is confirmed by numerous experiments.

3) Virtual charges have unique properties. They can "be born" and "disappear" under a certain influence, without fulfilling the law of conservation of charge.

4) Special interest is caused by unusual phenomena, in p-n transitions of semiconductor diodes, the description of which is absent in the scientific literature. They can be of great practical importance.

5) The problem of studying the physics of Tesla currents is new (Seifer, 2010). Experimental information is needed to create a theory (mathematical model) describing these phenomena. For this purpose it is necessary to develop new special measurement methods and new types of instruments.

6) As for the virtual charges themselves, the present information about them is not enough to give a description of their structure and the nature of the interaction with inertial charges and electromagnetic fields. It is necessary to carry out further experimental and theoretical studies (Angelov, 2016).

Note

Note 1. Such generators are usually used for surface hardening of steel.

\section{References}

Angelov, V. (2016). Two-Body Problem of Classical Electrodynamics with Radiation Terms - Energy Estimation (III) International Journal of Theoretical and Mathematical Physics, 6(2), 78-85.

Davis, J., \& Carr, J. (2002). Handbook radio engineer (M.: Dodeca-XXI).

Landau, L. D., \& Lifshitz, E. M. (2010). The Classical Theory of Fields (2nd ed.). Pergamon Press.

Panofsky, W., \& Phillips, M. (1969). Classical electricity and magnetism. Addison-Wesley World Student Series, Reading, Ma.: Addison-Wesley, 1969, 2nd ed.

Seifer, M. (2016). Wizard: The Life and Times of Nikola Tesla: Biography of a Genius (Citadel Press Kensington Publising Corp).

Zaev, N. (1994). Single-wire power line. Why do laws sleep? Inventor and innovator \#10/94 8-9. (in Russian)

Zaev, N. E., Avramenko, S. V., \& Lisin, V. N. (1991). The measuring of conduction current that is stimulated by polarization current. Journal of Russian Physical Society, (2), D1-2.

\section{Copyrights}

Copyright for this article is retained by the author(s), with first publication rights granted to the journal.

This is an open-access article distributed under the terms and conditions of the Creative Commons Attribution license (http://creativecommons.org/licenses/by/4.0/). 